\title{
High-Resolution Optical Observations of Interstellar Absorption Lines
}

\author{
Daniel E. Welty \\ University of Chicago, 5640 S. Ellis Ave., Chicago, IL 60637
}

\begin{abstract}
We briefly note several current topics concerning the properties of interstellar clouds for which high-resolution optical spectra play a significant role: (1) the recognition and characterization of small-scale (sub-pc) structure in both atomic and molecular gas; (2) the discovery of variations in the ${ }^{7} \mathrm{Li} /{ }^{6} \mathrm{Li}$ isotopic ratio in the nearby Galactic ISM; (3) the determination of atomic and molecular abundances and physical conditions for heavily reddened ("translucent") Galactic sightlines; and (4) studies of interstellar clouds in the LMC and SMC.
\end{abstract}

\section{Introduction}

High-resolution (FWHM $=0.3-1.5 \mathrm{~km} \mathrm{~s}^{-1}$ ) optical spectra of the interstellar (IS) absorption due to various atomic and molecular species have revealed complex velocity structure along many lines of sight (e.g., Crawford 1995; Crane, Lambert, \& Sheffer 1995; Welty, Morton, \& Hobbs 1996; Welty \& Hobbs 2001; Price et al. 2001). Fits to the line profiles suggest that the median line widths (FWHM) for individual components ("clouds") seen in the trace neutral species and the median separation between adjacent components are both less than 1.2 $\mathrm{km} \mathrm{s}^{-1}$. Moreover, the adjacent components can have very different properties (e.g., line width, Na I/Ca II ratio, molecular abundances). Such high resolution spectra are therefore necessary for both discerning and determining the composition and physical properties of individual IS clouds in different environments.

\section{Small-Scale Structure}

Pervasive small-scale (sub-pc) spatial structure in the ISM has been revealed both by differences in the line profiles of various species toward binary/multiple systems (e.g., Watson \& Meyer 1996; Lauroesch \& Meyer 1999; Pan, Federman, \& Welty 2001) and by temporal variations in the profiles in some sightlines (e.g., Blades et al. 1997; Price, Crawford, \& Barlow 2000; Crawford 2002; Lauroesch \& Meyer 2003). The physical characteristics of this seemingly ubiquitous structure are not well understood, however. For example, do the observed profile differences reflect differences in overall $N(\mathrm{H})$, in local density, in ionization, and/or in chemistry on those small scales? Toward the halo star HD 219188, where we have both multi-epoch high-resolution optical and UV spectra, the time-variable component is relatively $\operatorname{cool}(T<500 \mathrm{~K})$, with modest depletions, a relatively low density $n_{\mathrm{H}} \sim 25 \mathrm{~cm}^{-3}$, a low overall $N(\mathrm{H}) \sim 5 \times 10^{17} \mathrm{~cm}^{-2}$, and a relatively high fractional ionization $n_{e} / n_{\mathrm{H}} \gtrsim 0.03$ (Welty \& Fitzpatrick 2001). 


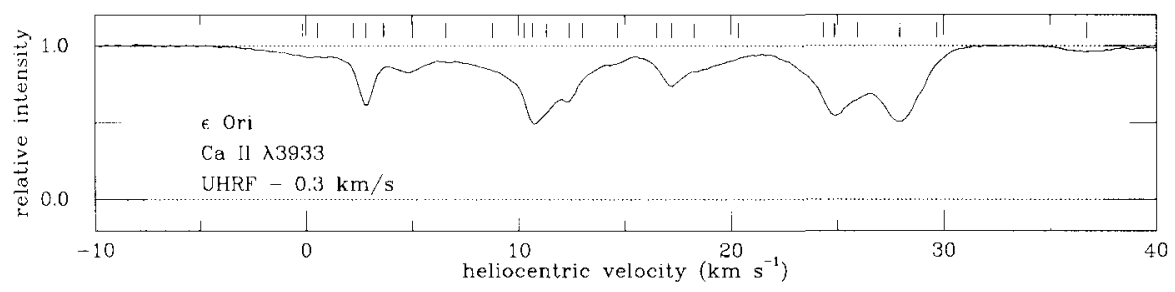

Figure 1. Complex interstellar Ca II absorption toward $\epsilon$ Ori.

In that particular case, the differences seem to be due to variations in density and ionization [rather than in overall $N(\mathrm{H})$ ] on scales of tens of $\mathrm{AU}$, without the large local pressures and densities sometimes inferred.

\section{Variations in ${ }^{7} \mathbf{L i} /{ }^{6} \mathbf{L i}$}

Abundances and isotope ratios for the light elements $\mathrm{Li}, \mathrm{Be}$, and $\mathrm{B}$ can provide information on big bang nucleosynthesis, Galactic chemical evolution, and cosmic ray spallation processes. Determination of the ${ }^{7} \mathrm{Li} /{ }^{6} \mathrm{Li}$ ratio requires optical spectra with both high S/N (the Li I doublet lines at $6708 \AA$ are very weak) and high spectral resolution (doublet separation $\sim 6.8 \mathrm{~km} \mathrm{~s}^{-1}$ for both, with the stronger member of the ${ }^{6} \mathrm{Li}$ I doublet almost coincident with the weaker member of the ${ }^{7} \mathrm{Li}$ I doublet; hyperfine structure in the ${ }^{7} \mathrm{Li}$ I lines). Knauth, Federman, $\&$ Lambert (2003) find interstellar ${ }^{7} \mathrm{Li} /{ }^{6} \mathrm{Li}$ ratios consistent with the solar system (meteoritic) value of 12.3 for four of the five sightlines with detected $\mathrm{Li}$ I absorption (see also Howarth et al. 2002). Toward o Per, however, fits to the observed line profile yield isotope ratios of 2.1 (close to the value expected for cosmic ray spallation) and 8.1 for the two components discernible in the spectra. While there are other indications that the cosmic ray flux might be enhanced in that direction, the resultant overall enhancement of $\mathrm{Li}$ is not observed.

\section{Properties of Translucent Clouds}

The so-called "translucent" IS clouds, with $1 \lesssim A_{V} \lesssim 5$, are thought to be intermediate (transitional?) objects between diffuse and dense clouds, and (as such) would provide useful tests for models of cloud chemistry. A survey of several dozen moderately reddened sightlines undertaken with FUSE, however, has not revealed the very high molecular fractions and more severe depletions expected for such objects (Rachford et al. 2002; Snow, Rachford, \& Figoski 2002). High resolution (0.3-1.8 $\left.\mathrm{km} \mathrm{s}^{-1}\right)$ optical spectra of Na I, K I, and CH absorption suggest that essentially all those sightlines contain multiple diffuse clouds (only), with no individual translucent clouds. Knowledge of the complex component structures in those sightlines provided by the optical spectra can be crucial for determining column densities for species such as HD and the excited rotational levels of $\mathrm{H}_{2}$, whose lines are observed at much lower resolution with FUSE (Snow et al. 2000; Ferlet et al. 2000). 


\section{ISM in the Magellanic Clouds}

Studies of the ISM in the Magellanic Clouds (MC) explore somewhat different environmental conditions from those typically probed in our Galaxy: lower metallicities, lower dust-to-gas ratios, stronger radiation fields, differences in UV extinction. Using the new "very long camera" on the coudé echelle spectrograph at the ESO $3.6 \mathrm{~m}$ telescope, we have obtained high-resolution $\left(1.2-2.0 \mathrm{~km} \mathrm{~s}^{-1}\right)$ spectra of IS Na I and Ca II toward SMC and LMC stars with $V \sim 11-13$ mag. These high-resolution spectra reveal numerous narrow, closely blended components in the MC ISM (similar to those seen in our own Galactic ISM). Together with UV spectra from IUE, HST, and FUSE, the optical spectra are being used to determine abundances, depletions, and physical conditions for diffuse clouds in the MC (Welty \& Hobbs 2001; Welty et al. 2001 and in prep.).

Acknowledgments. DEW acknowledges support from NASA LTSA grant NAG5-11413 to the University of Chicago. Slides for this contribution may be obtained from ftp://astro.uchicago.edu/pub/astro/welty/IAU25/iau25.ppt.

\section{References}

Blades, J. C., Sahu, M. S., He, L., Crawford, I. A., Barlow, M. J., \& Diego, F. 1997, ApJ, 478, 648

Crane, P., Lambert, D. L., \& Sheffer, Y. 1995, ApJS, 99, 107

Crawford, I. A. 1995, MNRAS, 277, 458

Crawford, I. A. 2002, MNRAS, 334, L33

Ferlet, R., et al. 2000, ApJ, 538, L69

Howarth, I. D., Price, R. J., Crawford, I. A., \& Hawkins, I. 2002, MNRAS, 335, 267

Knauth, D. C., Federman, S. R., \& Lambert, D. L. 2003, ApJ, 586, 268

Lauroesch, J. T. \& Meyer, D. M. 1999, ApJ, 519, L191

Lauroesch, J. T. \& Meyer, D. M. 2003, ApJ, 591, L123

Pan, K., Federman, S. R., \& Welty, D. E. 2001, ApJ, 558, L105

Price, R. J., Crawford, I. A., \& Barlow, M. J. 2000, MNRAS, 312, L43

Price, R. J., Crawford, I. A., Barlow, M. J., \& Howarth, I. D. 2001, MNRAS, 328, 555

Rachford, B. L., et al. 2002, ApJ, 577, 221

Snow, T. P., et al. 2000, ApJ, 538, L65

Snow, T. P., Rachford, B. L., \& Figoski, L. 2002, 573, 662

Watson, J. K., \& Meyer, D. M. 1996, ApJ, 473, L127

Welty, D. E., \& Fitzpatrick, E. L. 2001, ApJ, 551, L175

Welty, D. E., \& Hobbs, L. M. 2001, ApJS, 133, 345

Welty, D. E., Lauroesch, J. T., Blades, J. C., Hobbs, L. M., \& York, D. G. 2001, ApJ, $554, \mathrm{~L} 75$

Welty, D. E., Morton, D. C., \& Hobbs, L. M. 1996, ApJS, 106, 533 